\title{
A Screening Procedure on the Inhibitors of Substrate-incorporation in Tumor Cells Methodological Survey and Its Evaluation ${ }^{\dagger}$
}

\author{
Noboru ŌTAKE and Tetsuo SASAKI* \\ Institute of Applied Microbiology, University of Tokyo, \\ Bunkyo-ku, Tokyo 113, Japan \\ Received January 25, 1977
}

\begin{abstract}
A comparative study of inhibition on the substrate-incorporation in several species of tumor cells has been achieved in combination with the antibiotics having different action mechanisms. It was thus revealed that a large part of the antitumor antibiotics so far examined showed a marked inhibitory response even at a low concentration. Particularly, the antibiotics whose action mechanism were established primarily on cell membrane were markedly sensitive. An apparent difference in the sensitivity was observed in some groups of antitumor antibiotics which act on nucleic acid synthesis. A methodological survey together with an evaluation of this procedure is discussed.
\end{abstract}

The fact that many cancer therapeutic agents inhibit nucleic acid synthesis ${ }^{1)}$ prompts us to develope a newer screening method for its inhibitors. In the previous report, ${ }^{2)}$ we have developed a convenient procedure using ${ }^{3} \mathrm{H}$-uridine incorporation into Ehrlich ascites carcinoma cells to look for new antitumor antibiotics from microbial origin. We found that the labeled substrates were smoothly incorporated into intact tumor cells suspended in modified Hank's medium and that a satisfactory linear correlation of radioactivity increase was observed within three hours incubation.

Although this substrate-incorporation may be rationalized by turn-over rather than de novo synthesis, it was sensitive enough to be applied as a suitable screening procedure of the inhibitors.

Independently, Nitta et $a .^{3)}$ have reported a screening method using labeled amino acids incorporation into the homogenates of Ehrlich carcinoma cells to find out the inhibitors of protein synthesis. In view of the screening tactics, the use of living-cells having all of the

$\uparrow$ The Inhibitors of Substrate-incorporation in Tumor Cells. Part I.

* Present address: Central Research Laboratory, Nisshin Flour Milling Co., Ltd., Tsurugaoka, Ohimachi Iruma, Saitama, Japan. tumor characteristics is exceedingly charming and advantageous in order to discriminate the active compounds having a selective toxicity. In addition, for screening convenience, the development of a reliable and simpler procedure to discern an antitumor substances at a primary step from a multitude of natural products is earnestly expected.

For the sake to improve our procedure in practical screening program, a methodological survey as well as its evaluation are discussed.

In this report, we wish to present the results of a comparative study using three ordinary securable tumor cells of different origin, viz, Ehrlich ascites carcinoma, mouse lenkemia $\mathrm{L}-1210$ and human carcinoma KB in combination with the antibiotics having different action mechanisms.

\section{MATERIALS AND METHODS}

The following labeled substrates were purchased from Daiichi Pure Chemicals Co. Ltd, Tokyo, Japan; ${ }^{8} \mathrm{H}(\mathrm{G})$-uridine $(6.3 \mathrm{Ci} / \mathrm{mm}),\left(2-{ }^{14} \mathrm{C}\right)$-thymidine $(61 \mathrm{mCi})$ $\mathrm{mM}) \mathrm{L}-\left(1-{ }^{14} \mathrm{C}\right)$-leucine $(59 \mathrm{mCi} / \mathrm{mM})$.

The following antibiotics which were the authentic samples of our laboratory collection were used as model compounds through this work; penicillin $\mathrm{G},{ }^{* 1} \mathrm{O}$ carbamoyl-D-serine, D-cycloserine, bacitracin A, amphotericin $\mathrm{B}$, nystatin, valinomycin colistin $\mathrm{A},{ }^{* 2}$ monazo-

*1 The corresponding sodium salt was used.

*2 The corresponding sulfate was used. 
mycin, nigericin, ${ }^{* 1}$ lysocellin, ${ }^{* 1}$ nonactin, oligomycin $\mathrm{A}$, antimycin $\mathrm{A}$, rifampicin, chromomycin $\mathrm{A}_{3}$, daunomycin, actinomycin $D$, nogalamycin, triostin, pluramycin $A$, mitomycin $\mathrm{C}$, bleomycin $\mathrm{A}_{2},{ }^{* 3}$ tubercidin, cyclocitidine, cytosine arabinoside, chloramphenicol, streptomycin, ${ }^{* 2}$ erythromycin, tetracycline, puromycin, cycloheximide and blasticidin $\mathbf{S}$.

Microorganisms. Bacillus subtilis PCI 219, Escherichia coli NIHJ, Mycobacterium phlei 11D Timothee were used as test organism.

Tumor cells. Ehrlich ascites carcinoma cells were obtained from DDD mice inoculated with $10^{7}$ cells 8 days before. The cells were washed twice with saline and suspended in saline. Mouse leukemia L-1210 cells were prepared from DBA mice inoculated intraperitoneally with $10^{5}$ cells before 6 days. Human carcinoma $\mathrm{KB}$ cells were cultured in monolayer in Eeagle's minimal essential medium at $37^{\circ} \mathrm{C}$ and were harvested 7 days after inoculation.

The tumor cells were counted by Thomas cell counter and $10^{7}$ cells per $\mathrm{ml}$ of saline suspensions were prepared.

Determination of inhibitory ratio of labeled substrateincorporation. Fundamentally, the substrate-incorporation experiments were conducted in Hank's medium supplemented with $5 \%$ horse serum, $0.4 \%$ lactalbumin hydrolysate, except for the case of ${ }^{14} \mathrm{C}$-leucine, in which $0.1 \mathrm{mM}$ amino acids mixture minus leucine was substituted by lactalbumin hydrolysate.

In each run, $2 \times 10^{6}$ tumor cell were suspended in $0.8 \mathrm{ml}$ of the above medium contained ${ }^{3} \mathrm{H}$-uridine $(0.05 \mu \mathrm{Ci}),{ }^{14} \mathrm{C}$-thymidine $(0.10 \mu \mathrm{Ci})$ or ${ }^{14} \mathrm{C}$-leucine $(0.10 \mu \mathrm{Ci})$ and an adequate dose of test-antibiotic, respectively. A mixture was incubated at $37^{\circ} \mathrm{C}$ for $3 \mathrm{hr}$ with gentle shaking. After termination, an incubation mixture was cooled in an ice bath, diluted with equivolume of saline and the cells were collected on a membrane filter, successively washed with saline and $5 \%$ aqueous trichloroacetic acid. The labeled cells on the filter were dried and the radio activity were measured by a liquid scintilation counter (Beckman LS 230) using toluene scintillator (PPO $4 \mathrm{~g}$, POPOP $0.1 \mathrm{~g}$ in toluene $1000 \mathrm{ml})$.

Screening procedure for cultured broth. Substantial$\mathrm{ly}$, the same procedure described above was worked out for primary screening of the cultured broth using ${ }^{8} \mathrm{H}$ uridine and Ehrlich carcinoma cells. The filtered broth $(0.2 \mathrm{ml})$ which were obtained from three days cultures of unknown streptomyces newly isolated from soil were tested.

Spores from the slant cultures of streptomyces were inoculated directly into $100 \mathrm{ml}$ of a medium consisting

*8 The cupper chelate was used. of starch $3.0 \%$, polypeptone $1.0 \%$, meat extract $1.0 \%$, $\mathrm{CaCO}_{3} 0.2 \%, \mathrm{MgSO}_{4} \cdot 7 \mathrm{H}_{2} \mathrm{O} 0.1 \%, \mathrm{pH} 7,0$, in $500 \mathrm{ml}$ Ehlenmeyer flask, and cultured at $37^{\circ} \mathrm{C}$ on a rotary shaker.

Assay of antimicrobial activity. In parallel with the substrate-incorporation test, the cultured filtrates were simultaneously assayed their antibacterial activity by paper disk method against $B$. subtilis, $E$. coli and Myc, phlei.

Assay of antitumor activity in vivo. The cultured filtrates showed an activity more than $70 \%$ inhibition by substrate-incorporation test were assayed their antitumor activity. The DDD male mice $(20 \pm 1 \mathrm{~g})$ were inoculated intraperitoneally with $10^{7}$ cells of Ehrlich ascites carcinoma and treated daily by intraperitoneal administration of the filtrates $(0.2 \mathrm{ml}$ each) for 6 days starting $3 \mathrm{hr}$ after cell inoculation. Survival time and body weight were observed as criteria of tumor growth.

\section{RESULTS AND DISCUSSION}

\section{Patterns of substrate-incorporation in tumor cells}

At the outset, it was uncertain whether the tumor cells could smoothly incorporate the labeled substrates into their biosynthetic pathway of macromolecules. Preliminary investigation showed that both cells of Ehrlich ascites carcinoma and human carcinoma $\mathrm{KB}$ could incorporate ${ }^{14} \mathrm{C}$-thymidine, ${ }^{3} \mathrm{H}$-uridine and ${ }^{14} \mathrm{C}$-leucine in a similar pattern as shown in Fig. 1a and Fig. 1b, respectively. However, the cells of mouse leukemia L-1210 showed a rather weaker incorporation of these substrates, notably, in the case of ${ }^{14} \mathrm{C}$-thymidine which is presented in Fig. 1b. Probably, this may due to unstable nature of the tumor
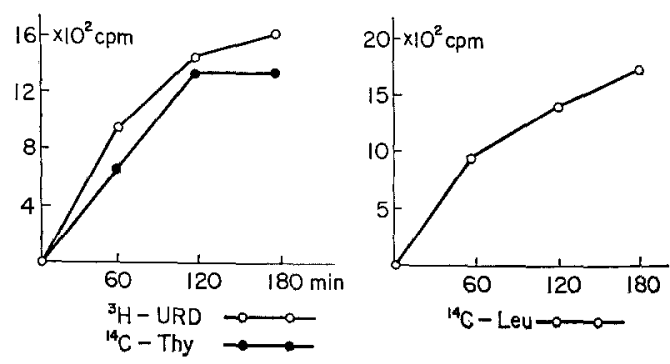

Fig. 1a. Patterns of Substrates Incorporation in Ehrlich Ascites Carcinoma Cells. 

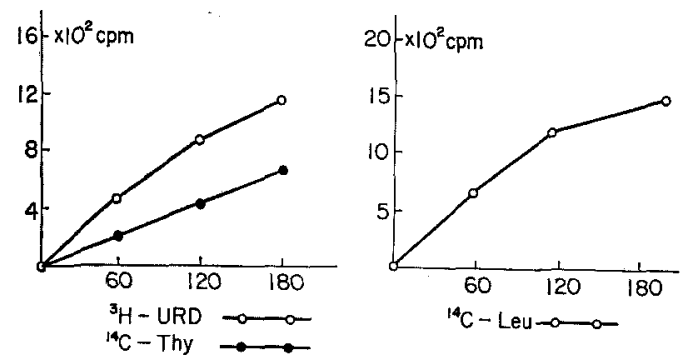

Fig. 1b. Patterns of Substrates Incorporation in Mouse Leukemia L-1210 Cells.
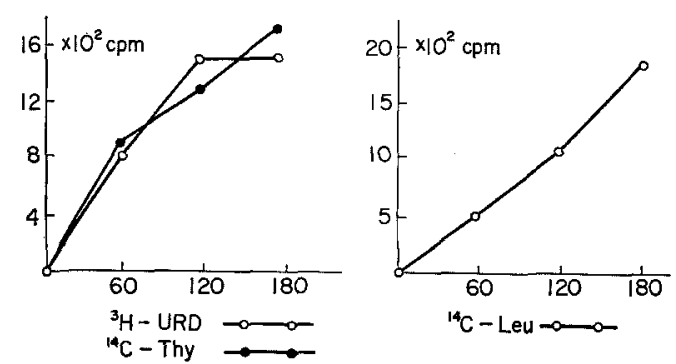

FIG. 1c. Patterns of Substrates Incorporation in Human Carcinoma KB Cells.

cells under the condition.

An approximate linear substrate-incorporation was observed in each pattern within 3 $\mathrm{hr}$ after incubation. In consequence the inhibition ratio $(\%)$ was defined by the following equation:

Inhibition ratio

$$
=\frac{\mathrm{cpm} \text { of control }-\mathrm{cpm} \text { of sample }}{\mathrm{cpm} \text { of control }} \times 100
$$

Inhibitory effects of the known antibiotics having different action mechanisms

The antibiotics and some synthetic compounds which were used as a model of inhibitors in this work were classified grossly into five groups by their action mechanisms according to Gale et al.," namely, those act on cell wall synthesis, on the function of cytoplasmic membrane, on energy-transfer enzymes, on nucleic acid synthesis and on ribosomal functions. In order to testify a dose-response of these model antibiotics in detail, each compound was examined triplicate at a different concentration and their effects were represented by inhibition ratio as summarized in Table I to Table V, respectively.

Inhibitors of cell wall synthesis

As would be expected that the antibiotics owe their action mechanism to inhibit cell wall synthesis are almost ineffective to the present assay systems.

Actually, penicillin G, ${ }^{4)}$ O-carbamoyl-Dserine $^{5)}$ and $\mathrm{D}$-cycloserine ${ }^{6)}$ showed almost no effect or less active even at a high concentration. Whereas, when at a high concentration, bacitracin $\mathrm{A}^{7}$ which affects cell wall synthesis also exerts a moderate inhibitory effect against incorporation of each substrate by each tumor cell.

\section{Antibiotics affecting the function of cytoplasmic membrane}

For convenience, the antibiotics which affect

Table I. Inhibitory Effect of the Antibiotics Affecting Cell Walls Synthesis ON THE Substrate-INCORPORATION IN TUMOR CElls

\begin{tabular}{|c|c|c|c|c|c|c|c|c|c|c|c|}
\hline & \multirow{2}{*}{ Antibiotics } & \multirow{2}{*}{$\begin{array}{c}\text { Concen- } \\
\text { tration } \\
\mu \mathrm{g} / \mathrm{ml}\end{array}$} & \multicolumn{3}{|c|}{${ }^{14} \mathrm{C}$-Thymidine } & \multicolumn{3}{|c|}{${ }^{3} \mathrm{H}$-Uridine } & \multicolumn{3}{|c|}{${ }^{14} \mathrm{C}$-Leucine } \\
\hline & & & Ehrilich & $L-120$ & $\mathrm{~KB}$ & Ehrlich & $L-1210$ & $\mathrm{~KB}$ & Ehrlich & $\mathrm{L}-1210$ & $\mathrm{~KB}$ \\
\hline 1 & Penicillin $\mathrm{G}$ & 166 & $10>$ & $10>$ & $10>$ & $10>$ & $10>$ & 13.2 & $10>$ & $10>$ & 16.5 \\
\hline 2 & $\begin{array}{l}\text { O-Carbomoyl- } \\
\text { D-serine }\end{array}$ & 166 & $10>$ & $10>$ & 19.3 & $10>$ & $10>$ & 12.1 & 18.9 & 16.3 & 11.7 \\
\hline 3 & Cycloserine & 166 & 42.4 & 17.1 & $10>$ & 34.2 & 12.4 & 20.3 & 13.8 & $10>$ & $10>$ \\
\hline 4 & Bacitracin & $\begin{array}{r}166 \\
33 \\
6.6\end{array}$ & $\begin{array}{l}89.4 \\
60.4 \\
20.2\end{array}$ & $\begin{array}{l}80.6 \\
54.7 \\
48.0\end{array}$ & $\begin{array}{l}86.3 \\
60.3 \\
17.4\end{array}$ & $\begin{array}{l}87.2 \\
66.3 \\
26.5\end{array}$ & $\begin{array}{l}77.7 \\
70.4 \\
60.3\end{array}$ & $\begin{array}{l}79.7 \\
58.4 \\
30.6\end{array}$ & $\begin{array}{l}84.7 \\
65.5 \\
40.4\end{array}$ & $\begin{array}{l}82.4 \\
72.9 \\
60.4\end{array}$ & $\begin{array}{l}79.9 \\
70.4 \\
43.3\end{array}$ \\
\hline
\end{tabular}

Abbreviation: Ehrlich, Ehrlich ascites carcinoma cells; L-1210, mouse leukemia L-1210 cells; $\mathrm{KB}$, human carcinoma KB cells. 
Table II. Inhibitory Effect of the Antibiotics Affecting Cell Membrane function on the Substrate-incorporation In Tumor Cells

\begin{tabular}{|c|c|c|c|c|c|c|c|c|c|c|c|}
\hline & \multirow{2}{*}{ Antibiotics } & \multirow{2}{*}{$\begin{array}{c}\text { Concen- } \\
\text { tration } \\
\mu \mathrm{g} / \mathrm{ml}\end{array}$} & \multicolumn{3}{|c|}{${ }^{14} \mathrm{C}$-Thymidine } & \multicolumn{3}{|c|}{${ }^{8} \mathrm{H}$-Uridine } & \multicolumn{3}{|c|}{${ }^{14} \mathrm{C}$-Leucine } \\
\hline & & & Ehrlich & $\mathrm{L}-1210$ & $\mathbf{K B}$ & Ehrlich & $\mathrm{L}-1210$ & $\mathrm{~KB}$ & Ehrlich & $\mathrm{L}-1210$ & KB \\
\hline \multirow[t]{3}{*}{1} & Amphothericine B & 3166 & 97.5 & 84.2 & 55.4 & 89.5 & 70.4 & 69.8 & 95,4 & 70.2 & 48.0 \\
\hline & & 33 & 97.0 & 28.5 & $10>$ & 89.8 & 62.8 & $10>$ & 96.7 & 17.3 & 13.2 \\
\hline & & 6.6 & 94.6 & 35.5 & $10>$ & 89.8 & 62.8 & $10>$ & 96.7 & 17.3 & 13.2 \\
\hline \multirow[t]{3}{*}{2} & Nystatin & 166 & 90.3 & 83.7 & 57.2 & 85.7 & 72.1 & 49.3 & 97.5 & 40.1 & 46.1 \\
\hline & & 33 & 62.8 & 56.2 & 12.9 & 61.8 & 40.5 & $10>$ & 97.7 & 19.3 & 12.4 \\
\hline & & 6.6 & 54.4 & 49.2 & $10>$ & 43.5 & 20,4 & $10>$ & 43.2 & $10>$ & $10>$ \\
\hline \multirow[t]{3}{*}{3} & Monazomycin & 166 & 94.1 & 84.0 & 96.7 & 93.3 & 89.4 & 99.2 & 99.0 & 83.6 & 88.3 \\
\hline & & 33 & 62.8 & 56.2 & 12.9 & 61.8 & 40.5 & $10>$ & 97.7 & 19.3 & 12.4 \\
\hline & & 6.6 & 11.2 & $10>$ & $10>$ & $10>$ & 10.4 & $10>$ & 43.2 & $10>$ & $10>$ \\
\hline \multirow[t]{3}{*}{4} & Nigericin & 166 & 96.4 & 84.4 & 96.7 & 93.3 & 89.4 & 99.2 & 99.0 & 83.6 & 88.3 \\
\hline & & 33 & 98.4 & 24.2 & 29.2 & 96.1 & 65.4 & 96.4 & 86.4 & 85.7 & 24.1 \\
\hline & & 6.6 & 91.1 & $10>$ & 16.5 & 90.6 & 54.4 & 32.3 & 85.3 & 70.9 & $10>$ \\
\hline \multirow[t]{3}{*}{5} & Lysocellin & 166 & 93.3 & 97.2 & 93.0 & 95.1 & 90.6 & 90.2 & 96.3 & 90.4 & 84.7 \\
\hline & & 33 & 92.1 & 83.3 & 83.0 & 94.5 & 81.8 & 82.2 & 92.8 & 80.3 & 65.0 \\
\hline & & 6.6 & 91.2 & 10.8 & 73.0 & 90.3 & 31.8 & 71.6 & 88.6 & 76.2 & 24.4 \\
\hline \multirow[t]{3}{*}{6} & Valinomycin & 166 & 70.4 & 81.1 & 69.2 & 63.2 & 48.6 & 54.4 & 49.9 & 40.4 & 50.2 \\
\hline & & 33 & 43.8 & 49.2 & 60.4 & 40.4 & 30.3 & 39.2 & 38.4 & 19.8 & 17.3 \\
\hline & & 6.6 & $10>$ & $10>$ & 12.8 & $10>$ & 19.3 & $10>$ & $10>$ & $10>$ & $10>$ \\
\hline \multirow[t]{3}{*}{7} & Nonactin & 166 & 98.0 & 97.4 & 99.4 & 99.0 & 96.1 & 94.4 & 93.8 & 92.2 & 90.4 \\
\hline & & 33 & 74.2 & 76.4 & 24.2 & 79.2 & 75.0 & 64.0 & 80.5 & 70.5 & 69.6 \\
\hline & & 6.6 & $10>$ & $10>$ & $10>$ & $10>$ & $10>$ & $10>$ & $10>$ & $10>$ & $10>$ \\
\hline \multirow{2}{*}{8} & Colistin & 160 & 48.4 & 56.3 & 33.6 & $10>$ & $10>$ & $10>$ & 28.0 & 23.4 & 26.4 \\
\hline & & 6.6 & $10>$ & 12.6 & $\begin{array}{l}50.1 \\
10>\end{array}$ & $10>$ & $\begin{array}{l}10> \\
10>\end{array}$ & $\begin{array}{l}10> \\
10>\end{array}$ & $\begin{array}{l}12.4 \\
10>\end{array}$ & 10.3 & 20.0 \\
\hline
\end{tabular}

Abbreviation: Ehrlich, Ehrlich ascites carcinoma cells; L-1210, mouse leukemia L-1210 cells; $\mathrm{KB}$, human carcinoma KB cells.

the function of cytoplasmic membrane were divided into four subgroups according to their structural characteristic and molecular basis of action, namely, the polyene macrolides, the cyclic peptides, the polyethers and the macrotetralides. Accordingly, some typical members of each subgroup were chosen as model compounds to examine their inhibitory effect on this assay system. The results are given in Table II.

At the first, amphotericin $\mathbf{B}^{8)}$ and nystatin ${ }^{9)}$ which belong to the typical polyene macrolides were known as the agents to alter the permeability of membrane. Actually, these two antibiotics showed a remarkable inhibitory effect against each substrate by Ehrlich tumor cells, but did moderately by $\mathrm{L}-1210$ leukemia cells and only weakly by human $\mathrm{KB}$ cells.

Valinomycin $^{103}$ and monazomycin ${ }^{11}$ which were characterized as the cation-ionophore showed a distinct dose-response effect depending on the concentration of the antibiotics. It is noteceable that these two antibiotics were almost inactive at a lower concentration of $6 \mathrm{mcg} / \mathrm{ml}$.

Colistin $\mathrm{A}^{12)}$ which, however, represents the another type of cyclic peptides showed only a weak effect at a higher concentration against ${ }^{14} \mathrm{C}$-thymidine incorporation.

On the other hand, nigericin ${ }^{13)}$ and lysocellin, ${ }^{14)}$ the powerful cation-ionophores exerted a non-specific inhibitory effect against incorporation of each substrate, nevertheless, a slight difference of sensitivity was observed with regard to the origin of tumor cell, e.g., those of leukemia L-1210 and human KB were less sensitive at a low concentration.

The last model compound was nonactin ${ }^{151}$ which was known as an uncoupler as well as an ionophore also exhibited a non-specific 
TABle III. InHIBItory EfFect of the InHIBItors of ENERgy Transfer ENZymes ON THE SUbSTRATE-INCORPORATION IN TUMOR CELlS

\begin{tabular}{|c|c|c|c|c|c|c|c|c|c|c|c|}
\hline & \multirow{2}{*}{ Antibiotics } & \multirow{2}{*}{$\begin{array}{c}\text { Concen- } \\
\text { tration } \\
\mu \mathrm{g} / \mathrm{ml}\end{array}$} & \multicolumn{3}{|c|}{${ }^{14} \mathrm{C}$-Thymidine } & \multicolumn{3}{|c|}{${ }^{8} \mathrm{H}$-Uridine } & \multicolumn{3}{|c|}{${ }^{14} \mathrm{C}$-Leucine } \\
\hline & & & Ehrlich & $\mathrm{L}-1210$ & $\mathrm{~KB}$ & Ehrlich & $L-1210$ & $\mathrm{~KB}$ & Ehrlich & $\mathrm{L}-1210$ & $\mathrm{~KB}$ \\
\hline \multirow[t]{3}{*}{1} & Oligomycin & 166 & 77.8 & 25.1 & 72.3 & 71.3 & 80.6 & 93.4 & 88.6 & 67.0 & 80.4 \\
\hline & & 33 & 57.9 & $10>$ & 43.8 & 62.4 & 75.3 & 80.8 & 76.2 & 65.0 & 83.7 \\
\hline & & 6.6 & 37.4 & $10>$ & $10>$ & 45.3 & 48.4 & 63.4 & 49.8 & 50.4 & 65.5 \\
\hline \multirow[t]{3}{*}{2} & Antimycin A & 166 & 94.2 & 92.1 & 95.7 & 97.0 & 98.4 & 99.3 & 90.1 & 84.3 & 88.9 \\
\hline & & 33 & 84.3 & 70.3 & 66.8 & 69.6 & 83.4 & 75.9 & 86.1 & 71.6 & 54.6 \\
\hline & & 6.6 & 72.1 & 24.3 & 32.1 & 47.4 & 51.2 & 45.6 & 27.2 & $10>$ & 39.5 \\
\hline
\end{tabular}

Abbreviation: Ehrlich, Ehrlich ascites carcinoma cells; L-1210, mouse leukemia L-1210 cells; $\mathrm{KB}$, human carcinoma $\mathrm{KB}$ cells.

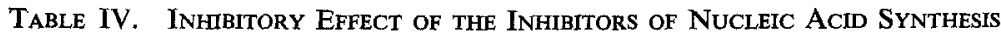
on the Substrate-incorporation in Tumor Cells

\begin{tabular}{|c|c|c|c|c|c|c|c|c|c|c|c|}
\hline & \multirow{2}{*}{ Antibiotics } & \multirow{2}{*}{$\begin{array}{c}\text { Concen- } \\
\text { tration } \\
\mu \mathrm{g} / \mathrm{ml}\end{array}$} & \multicolumn{3}{|c|}{${ }^{14} \mathrm{C}-\mathrm{Thymidine}$} & \multicolumn{3}{|c|}{${ }^{3} \mathrm{H}$-Uridine } & \multicolumn{3}{|c|}{${ }^{14} \mathrm{C}$-Leucine } \\
\hline & & & Ehrlich & $\mathrm{L}-1210$ & $\mathrm{~KB}$ & Ehrlich & $L-1210$ & $\mathbf{K B}$ & Ehrlich & $\mathrm{L}-1210$ & $\mathbf{K B}$ \\
\hline \multirow[t]{3}{*}{1} & \multirow[t]{3}{*}{ Actinomycin-D } & 16.6 & 93.3 & 70.5 & 86.0 & 93.8 & 85.6 & 68.4 & 89.4 & 48.5 & 78.7 \\
\hline & & 3.3 & $10>$ & $10>$ & 79.4 & 59.6 & 69.6 & 85.3 & 70.0 & 43.8 & 27.4 \\
\hline & & 0.66 & $10>$ & 13.1 & 38.5 & 51.2 & 67.8 & 73.4 & 33.9 & 45.2 & 27.4 \\
\hline \multirow[t]{3}{*}{2} & \multirow[t]{3}{*}{ Chromomycin- $\mathrm{A}_{3}$} & 316.6 & 51.5 & 53.8 & 85.1 & 66.0 & 49.0 & 86.4 & 42.4 & 44.8 & 27.8 \\
\hline & & 3.3 & 43.5 & 49.7 & 85.2 & 62.5 & $10>$ & 79.9 & 41.6 & 33.2 & 16.9 \\
\hline & & 0.66 & 11.9 & 13.8 & 81.9 & 40.9 & $10>$ & 48.3 & 17.4 & 13.2 & $10>$ \\
\hline \multirow[t]{2}{*}{3} & \multirow{2}{*}{ Daunomycin } & 16.6 & 62.3 & 85.2 & 94.6 & 64.2 & 81.7 & 90.6 & 23.8 & 76.7 & 25.4 \\
\hline & & 3.3 & 47.2 & 61.3 & 82.0 & 50.3 & 41.9 & 83.4 & 17.3 & 13.2 & $10>$ \\
\hline \multirow[t]{3}{*}{4} & \multirow[t]{3}{*}{ Nogalamycin } & 16.6 & 95.1 & 92.5 & 96.4 & 90.3 & 92.1 & 91.3 & 65.8 & 30.3 & 45.2 \\
\hline & & 3.3 & 94.2 & 74.7 & 91.3 & 92.1 & 86.3 & 93.9 & 54.3 & 31.1 & 28.8 \\
\hline & & 0.66 & 90.7 & 63.2 & 32.1 & 89.4 & 84.2 & 60.8 & 39.2 & 26.4 & 19.1 \\
\hline \multirow[t]{3}{*}{5} & \multirow[t]{3}{*}{ Triostin } & 16.6 & 89.3 & 74.2 & $10>$ & 92.1 & 89.4 & 73.6 & 54.3 & 47.2 & 61.6 \\
\hline & & 3.3 & 83.1 & 24.6 & 20.0 & 80.1 & 35.1 & 17.9 & 28.1 & 34.0 & 30.3 \\
\hline & & 0.66 & 40.3 & 17.6 & 12.5 & $10>$ & 31.6 & 15.7 & 13.2 & 18.4 & 50.2 \\
\hline \multirow[t]{3}{*}{6} & \multirow[t]{3}{*}{ Pluramycin-A } & 16.6 & 99.0 & 11.8 & 99.0 & 99.0 & 73.2 & 93.0 & 93.8 & 33.2 & 88.0 \\
\hline & & 3.3 & 99.0 & 19.5 & 77.8 & 95.4 & 64.0 & 93.0 & 29.4 & 29.4 & 61.0 \\
\hline & & 0.66 & 98.8 & 17.1 & 96.9 & 92.1 & 52.3 & 93.7 & 18.5 & 18.5 & 49.1 \\
\hline \multirow[t]{3}{*}{7} & \multirow[t]{3}{*}{ Bleomycin $\mathbf{A}_{2}$} & 166 & 41.3 & 72.7 & 74.3 & 23.0 & 69.3 & 67.5 & 24.3 & 24.4 & 28.6 \\
\hline & & 33 & 36.6 & 63.2 & 64.3 & 16.9 & 58.2 & 44.9 & 17.6 & 16.4 & 40.2 \\
\hline & & 6.6 & $10>$ & 24.8 & 43.2 & $10>$ & 13.4 & 10.1 & 16.3 & 15.2 & 17.3 \\
\hline \multirow[t]{3}{*}{8} & \multirow[t]{3}{*}{ Tubercidin } & 166 & 24.0 & 60.2 & 82.5 & 40.4 & 53.8 & 78.2 & 37.2 & 40.3 & 69.8 \\
\hline & & 33 & 22.3 & 39.4 & 85.5 & 23.2 & 40.3 & 86.0 & 21.3 & 28.2 & 61.0 \\
\hline & & 6.6 & 13.8 & 20.1 & 85.0 & 10.3 & 11.7 & 80.7 & 29.3 & 12.4 & $10>$ \\
\hline \multirow[t]{3}{*}{9} & \multirow[t]{3}{*}{ Mitomycin-C } & 166 & 89.4 & 80.6 & 86.3 & 87.2 & 77.7 & 79.7 & 84.7 & 82.2 & 79.9 \\
\hline & & 33 & 60.4 & 54.7 & 60.3 & 66.3 & 70.4 & 58.4 & 65.5 & 72.4 & 70.4 \\
\hline & & 6.6 & 20.2 & 48.0 & 17.4 & 26.5 & 60.3 & 30.6 & 40.4 & 60.4 & 43.3 \\
\hline \multirow[t]{2}{*}{10} & Cyclocytidine & 166 & 93.0 & 90.1 & 92.8 & 38.3 & 25.9 & 63.4 & 29.3 & 12.4 & 10.7 \\
\hline & & 33 & 94.2 & 83.4 & 87.4 & 17.4 & 20.3 & 67.4 & 17.2 & 10.7 & $10>$ \\
\hline 11 & Cytidine- & 166 & 96.6 & 83.3 & 93.2 & 42.8 & 38.5 & 17.2 & 24.3 & 19.2 & 17.1 \\
\hline & arabinoside & 33 & 96.3 & 90.3 & 80.8 & 24.5 & 21.3 & 20.1 & 17.5 & 16.4 & $10>$ \\
\hline 12 & Rifampicin & 166 & $10>$ & $10>$ & 23.0 & $10>$ & $10>$ & 34.7 & 35.8 & 13.2 & 24.2 \\
\hline & & 33 & $10>$ & $10>$ & $10>$ & $10>$ & $10>$ & $10>$ & 24.1 & 12.0 & 15.3 \\
\hline
\end{tabular}

Abbreviation: Ehrlich, Ehrlich ascites carcinoma cells; L-1210, mouse leukemia L-1210 cells; $\mathrm{KB}$, human carcinoma KB cells. 
effect.

Conclusively, a large number of the active compounds affecting the function of cell membrane exhibited a paralleled response on the substrates incorporation as well as antimicrobial activity.

\section{Inhibitors of energy transfer enzymes}

Oligomycin $\mathrm{A}^{16)}$ and antimycin $\mathrm{A}^{17)}$ are known as the inhibitors of energy transfer enzymes or of respiratory chains, respectively. Both antibiotics exerted a distinct inhibitory effect in each tumor cells as shown in Table III. This implies that an analogous active compound of this class may also be screened with a great possibility.

\section{Inhibitors of nucleic acid synthesis}

Originally this screening procedure was developed with the aim of finding new inhibitors of nucleic acid synthesis. Hitherto, almost all of the antibiotics whose molecular basis of action were established as agents affecting DNA or RNA functions or the enzyme systems responsible for their synthesis have been demonstrated in parallel as the potential antitumor substances. Reasonably, these antibiotics can be roughly classified into three subgroups based on their mode of action; 1) inhibitors of nucleic acid synthesis by binding with responsible enzyme systems, 2) intercalculating or strand-breaking agents of DNA, 3) structural analogs of nucleic acid components.

The results of this group are compared in Table IV.

The first subgroup contains rifampicin, ${ }^{18)}$ chromomycin $\mathrm{A}_{3}{ }^{19)}$ and daunomycin ${ }^{20)}$ which, except for rifampicin showed a moderate sensitivity of inhibition on the incorporation of ${ }^{3} \mathrm{H}$-uridine and ${ }^{14} \mathrm{C}$-thymidine in each tumor cells, however, as a matter of course, they were markedly less active against ${ }^{14} \mathrm{C}$-leucine incorporation. Rifampicin was extremely inactive in each substrates even at a higher concentration of $166 \mathrm{mcg} / \mathrm{ml}$.

The second subgroup contains actinomycin $\mathrm{D},{ }^{21)}$ nogalamycin, ${ }^{22)}$ triostin, ${ }^{23)}$ pluramycin
$\mathrm{A}^{24)}$ mitomycin $\mathrm{C}^{25)}$ and bleomycin $\mathrm{A}_{2}{ }^{23)}$ which belong to the well-established members of antibiotics affecting the template function of DNA. Of these, actinomycin D, nogalamycin, pluramycin $A$, triostin and mitomycin $C$ exerted a non-specific and strong inhibitory effect on ${ }^{3} \mathrm{H}$-uridine and ${ }^{14} \mathrm{C}$-thymidine incorporation, but were less active on the incorporation of ${ }^{14} \mathrm{C}$-leucine as would be expected beforehand.

It is of interest as well as surprise that bleomycin $A_{2}$ showed a rather weak response in general against the incorporation of each substrate even at a high concentration. However, the cells of L-1210 leukemia and human KB showed a considerable sensitivity than those of Ehrlich carcinoma.

The third subgroup of agents which act by substituting for natural nucleosides in nucleic acids includes tubercidin ${ }^{27)}$ and two synthetic structural analogs, viz, cyclocytidine ${ }^{28\}}$ and cytosine arabinoside. ${ }^{291}$

It is noteworthy that tubercidin exerted a marked selectivity toward the species of tumorcells, namely, only human KB cells were susceptible to this unusual nucleoside. It is very reasonable of course that the two synthetic structural analogs showed a rather intensive effect on ${ }^{14} \mathrm{C}$-thymidine incorporation than the other substrates.

\section{Antibiotic inhibitors of ribosomal function}

The antibiotics which exert their inhibitory effect on protein synthesis contain a number of the clinically important drugs including chloramphenicol, ${ }^{30)}$ streptomycin, ${ }^{31)}$ erythromycin ${ }^{32)}$ and tetracycline. ${ }^{33)}$ In general, these antibiotics show a selective toxicity against bacteria but not against eukaryotic and mammalian cells. In contrast, puromycin, ${ }^{34)}$ cycloheximide, ${ }^{35}$ ? blasticidin $\mathrm{S}^{36)}$ and amicetin ${ }^{37)}$ were known as less selective inhibitors of ribosomal function.

The results of inhibition examined on these model compounds are summarized in Table V.

As would be expected in common that the ${ }^{14} \mathrm{C}$-leucine incorporation might be extensively inhibited by these antibiotics, but, in fact, only puromycin and cycloheximide showed a con- 
TABLE V. INHIBITORY EFFECT OF THE INHIBITORS OF Ribosomal Function on the Substrate-InCorporation In Tumor Cells

\begin{tabular}{|c|c|c|c|c|c|c|c|c|c|c|c|}
\hline & \multirow{2}{*}{ Antibiotics } & \multirow{2}{*}{$\begin{array}{c}\text { Concen- } \\
\text { tration } \\
\mu \mathrm{g} / \mathrm{ml}\end{array}$} & \multicolumn{3}{|c|}{${ }^{14} \mathrm{C}-$ Thymidine } & \multicolumn{3}{|c|}{${ }^{8} \mathrm{H}$-Uridine } & \multicolumn{3}{|c|}{${ }^{14} \mathrm{C}$-Leucine } \\
\hline & & & Ehrlich & $L-1210$ & KB & Ehrlich & $\mathrm{L}-1210$ & $\mathrm{~KB}$ & Ehrlich & $L-1210$ & $\mathrm{~KB}$ \\
\hline \multirow[t]{2}{*}{1} & Puromycin & 33.3 & 10 & 10 & 85.8 & 16.6 & 10 & 73.0 & 93.2 & 91.0 & 92.9 \\
\hline & & 6.6 & 10 & 10 & 79.2 & 10 & 10 & 64.8 & 94.0 & 66.5 & 80.3 \\
\hline \multirow[t]{3}{*}{2} & Amicetin & 166 & 32.5 & 31.4 & 71.7 & 37.5 & 17.2 & 72.4 & 78.0 & 67.3 & 74.2 \\
\hline & & 33.3 & 19.2 & 19.3 & 69.4 & 16.3 & 20.3 & 77.9 & 40.0 & 43.2 & 26.4 \\
\hline & & 6.6 & 17.2 & 20.1 & 63.2 & 11.4 & 12.4 & 70.3 & 30.2 & 29.3 & 18.4 \\
\hline \multirow[t]{2}{*}{3} & Blasticidin S & 166 & 20.3 & 24.3 & 67.0 & 10 & 10 & 63.9 & 59.4 & 31.4 & 71.8 \\
\hline & & 33 & 10 & 10 & 53.4 & 10 & 16.0 & 61.9 & 38.3 & 21.6 & 35.1 \\
\hline 4 & Streptomycin & 166 & 10 & 10 & 10 & 10 & 10 & 10 & 10 & 10 & 10 \\
\hline \multirow[t]{3}{*}{5} & Cycloheximide & 166 & 64.3 & 69.2 & 68.3 & 14.3 & 10 & 10 & 84.9 & 90.4 & 88.6 \\
\hline & & 33.3 & 38.3 & 23.1 & 42.7 & 10 & 10 & 10 & 83.7 & 88.0 & 87.2 \\
\hline & & 6.6 & 10 & 18.4 & 20.4 & 10 & 10 & 10 & 82.8 & 80.8 & 84.1 \\
\hline \multirow[t]{3}{*}{6} & Tetracycline & 166 & 50.3 & 26.4 & 40.1 & 27.2 & 20.3 & 19.8 & 62.4 & 40.4 & 38.7 \\
\hline & & 33.3 & 24.0 & 20.0 & 13.8 & 20.4 & 19.4 & 14.2 & 47.4 & 24.3 & 19.2 \\
\hline & & 6.6 & 10 & 10 & 10 & 10 & 10 & 10 & 17.2 & 24.3 & 19.2 \\
\hline \multirow[t]{3}{*}{7} & Chrolam- & 166 & 60.4 & 38.7 & 29.4 & 39.2 & 30.3 & 20.2 & 68.2 & 43.2 & 51.4 \\
\hline & phenicol & 33.3 & 11.1 & 24.1 & 10 & 17.2 & 19.4 & 12.8 & 34.3 & 40.2 & 29.6 \\
\hline & & 6.6 & 10 & 10 & 10 & 10 & 10 & 10 & 12.1 & 17.4 & 10 \\
\hline \multirow[t]{3}{*}{8} & Erythromycin & 166 & 24.2 & 10 & 38.2 & 28.2 & 29.4 & 33.2 & 52.6 & 26.9 & 30.3 \\
\hline & & 33.3 & 18.4 & 10 & 10 & 17.4 & 10 & 29.6 & 24.6 & 10 & 17.2 \\
\hline & & 6.6 & 10 & 10 & 10 & 10 & 10 & 10 & 10 & 10 & 10 \\
\hline
\end{tabular}

Abbreviation: Ehrlich, Ehrlich ascites carcinoma cells; L-1210, mouse leukemia L-1210 cells; $\mathrm{KB}$, human carcinoma $\mathrm{KB}$ cells.

siderable effect. Surprisingly, streptomycin was almost devoid of its inhibitory effect regardless the species of tumor-cells and substrates.

It is noteceable that, the inhibition patterns of these antibiotics showed a distinct selectivity both in the species of tumor cells and the kind of substrates. For exemple, the human carcinoma $\mathrm{KB}$ cells showed a more significant sensitivity than those of Ehrlich carcinoma and leukemia $\mathrm{L}-1210$ in significant incorporation of ${ }^{14} \mathrm{C}$-thymidine and ${ }^{3} \mathrm{H}$-uridine.

Accordingly, the inhibitors of this group showed a large variety of response depending on the molecular basis of the individual compounds as compared in Table $\mathrm{V}$.

Survey and evaluation of this screening procedure

On the basis of the results so far discussed, it should be emphasized here that this screening procedure is valuable as well as promising in view of a screening convenience and efficacy.

In particular, it seems extremely favorable to use the living cells because there are furnished with the whole enzyme systems which are responsible to essential metabolism. Actually, with the use of this procedure in practical screening program, we have resulted in a discovery of an active substance belonging to a new class of antitumor substances, which act directly on the breakdown of cell membranes as will be demonstrated in the following report. ${ }^{383}$

On the contrary, it should be pointed out some defective profile of this procedure so far as accounted for.

Primarily, this procedure was not sensitive enough against some types of model antibiotics, in particular, those belong to the ansamycin antibiotics. Likewise it was almost inactive against bleomycin antibiotics.

On the contrary, a given group of antibiotics such as equinomycins, antimycins, xanthomycin and actinomycins which showed an excessive sensitivity even at a lower concentration would be screened with a great 
frequency by this procedure.

\section{Results of a practical screening program}

Since our primary concern is looking for a new antitumor substance from microbial origin, we have planned to evaluate this procedure by a screening program in practice against a limited number of streptomyces. For convenience, a combination of ${ }^{3} \mathrm{H}$-uridine and Ehrlich ascites carcinoma cells was selected as a screening system. As a result, among 560 strains of streptomyces so far tested, 55 cultured filtrates showed above $70 \%$ inhibition of labeled substrate-incorporation. Subsequent in vivo assay against Ehrlich ascites carcinoma in mouse, 5 out 55 of these cultured filtrates exhibited a significant paralleled antitumor activity.

After careful investigation of the active principles, it has resulted in the identification of four known products, e.g., echinomycin A, actinomycin $\mathrm{D}$, antimycin $\mathrm{A}$ and rhodomycin.

However, a remaining strain whose cultured broth did not exhibit any antimicrobial activity, exerted a considerable antitumor activity. Eventually, this active principle was designated renastacarcin and its isolation, characterization and biological properties will be reported in the subsequent papers as a part of this series.

On the basis of the results so far discussed on the model experiments along with those of the practical screening program, we can conclude reasonably that the present "substrateincorporation procedure" is a valuable and convenient method for a primary screening of the active substances having antitumor activity.

Acknowledgement. The authors are grateful to Professor H. Yonehara of the Institute of Applied Microbiology, University of Tokyo for his valuable and keen discussions through the work. This work is supported in whole by a Grant of the Ministry of Education, Culture and Science, the Government of Japan.

\section{REFERENCES}

1) I. H. Goldberg and P. A. Friedman, Ann. Rev.
Biochem., 40, 775 (1971).

2) T. Tanaka, K. Sakaguchi, N. Ôtake and H. Yonehara, Agric. Biol. Chem., 32, 100 (1968).

3) K. Nitta, S. Mizuno and H. Umezawa, J. Antibiot. Ser. A, 19, 282 (1966).

4) E. F. Gale, E. Cundliffe, P. E. Reynolds, M. H. Richmond and M. J. Warning, "The Molecular Basis of Antibiotic Action," John Wiley \& Sons Ltd., 1972.

5) J. L. Lynch and F. C. Neuhaus, J. Bacteriol., 91, 449 (1966).

6) F. C. Neuhans, Antimicrob. Agents Chemothr., 1967, p. 304.

7) K. J. Stone and J. L. Strominger, Proc. Natl. Acad. Sci. U.S.A., 68, 3223 (1971).

8) J. O. Lampen, J. W. Gill, P. M. Arnow and L. Magnaplaza, J. Bact., 86, 845 (1963).

9) S.C. Kinsky, J. Haxby, C.B. Kinsky, R.A. Demel and L. L. M. Van Deenen, Biochim. Biophys. Acta, 152, 174 (1968).

10) V. T. Ivanov, I. A. Laine, N. D. Senyvina, L. B. Popov, E. M. Ovchinnikov, A. Yu and M. M. Shemyakin, Biochem. Biophys. Res. Commun., 34, 803 (1969).

11) H. A. Laroy, S. N. Graven and S. Estradao, Fed. Proc., 26, 1355 (1967).

12) J. Wahn, G. Lutsch, T. Rockstoh and K. Zapf, Arch. Microbiol., 27, 1263 (1968).

13) M. Pinkerton and L. K. Steinrauf, J. Mol. Biol., 49, 533 (1970).

14) M. Mitani and T. Yamanishi, E. Ebata, M. Koenuma and N. Ötake, J. Antibiot, in press.

15) B. T. Kilbourn, J. D. Dunitz, L. A. R. Piodo and W. Simon, J. Mol. Biol., 30, 559 (1967).

16) B. Bulos and E. Racker, J. Biol. Chem., 243, 3891 (1968).

17) F. L. Crane, Y. Hatefi, L. Lester and C. Widmer, Biochim. Biophys. Acta, 25, 220 (1957).

18) G. Hartman, K. O. Honikel, F. Kniisel and J. Nuesch, ibid., 145, 843 (1967).

19) W. Kersten and H. Kersten, "Molecular association in biology," Academic Press, London, 1968, p. 289.

20) B. K. Bhuyan and C. G. Smith, Proc. Natl. Acad. Sci. U.S.A, 54, 566 (1965).

21) E. Reich and I. H. Goldbelg, Prog. Nucleic Acid Res. and Mol. Biol., 3, 183 (1964).

22) A. Sentenac, A. Ruet and P. Fromageot, Eur. J. Biochem., 5, 385 (1968).

23) A. Sentenac, E. J. Simon and P. Fromageot, Biochim. Biophys. Acta, 161, 299 (1968).

24) N. Tanaka, N. Nagai, H. Yamaguchi and H. Umezawa, Biochem. Biophys. Res. Commun., 21, 328 (1965).

25) W. Szybalsky and V. N. Iyer, Fed. Proc., 23, 946 (1964).

26) H. Suzuki, K. Nagai, H. Yamaki, N. Tanaka and 
H. Umezawa, J. Antibiot, 21, 379 (1969).

27) D. C. Ward and E. Reich, Ann. Rep. Med. Chem., 1970, p. 272.

28) P. Roy-Burman, Y. H. Hung and D. W. Visser, Biochem. Biophys. Res. Commun., 42, 445 (1971).

29) P. Roy-Burman, Recent Results Cancer Res., 25, 36 (1970). Springer Verlag, Heidelbelg.

30) D. Vazquez, "Antibiotics," Vol. 1, ed. by D. Gottlieb and P. D. Shaw, Springer-Verlag, New York, 1967, pp. 366, 387.

31) J. Modolell and B. D. Davis, Proc. Natl. Acad. Sci. U.S.A., 61, 1729 (1968); ibid., 67, 1148 (1970); ibid., 68, 1796 (1971).

32) J. C. H. Mao and E. E. Robishaw, Biochim. Bio- phys. Acta, 23, 857 (1971); idem, Biochem., 10, 2054 (1971).

33) E. Cundiffe, "Molecular Mechanisms of Antibiotic Action on Protein Biosynthesis and Membranes," ed. by E. Munoz, F. Ferrandiz and D. Vazqnez, Elservier, Amsterdam, 1972.

34) D. Nathans, Fed. Proc., 23, 984 (1964).

35) D. S. Beattie, FEBS Lett., 9, 232 (1970).

36) J. M. Clark, "Antibiotics," ed. by D. Gottlieb and P.D. Shaw, Vol. 1, Springer-Verlag, New York, 1967, p. 278.

37) F. N. Chang and J. G. Flaks, Proc. Natl. Acad. Sci. U.S.A., 67, 1321 (1970).

38) The subsequent papers. 